\title{
Application of a score system to evaluate the risk of malnutrition in a multiple hospital setting
}

\author{
Maria Immacolata Spagnuolo, Ilaria Liguoro, Fabrizia Chiatto, Daniela Mambretti and Alfredo Guarino*
}

\begin{abstract}
Background: An increased but unpredictable risk of malnutrition is associated with hospitalization, especially in children with chronic diseases. We investigated the applicability of Screening Tool for Risk of Impaired Nutritional Status and Growth (STRONGkids), an instrument proposed to estimate the risk of malnutrition in hospitalized children. We also evaluated the role of age and co-morbidities as risk for malnutrition.

Methods: The STRONGkids consists of 4 items providing a score that classifies a patient in low, moderate, high risk for malnutrition. A prospective observational multi-centre study was performed in 12 Italian hospitals. Children 1-18 years consecutively admitted and otherwise unselected were enrolled. Their STRONGkids score was obtained and compared with the actual nutritional status expressed as BMI and Height for Age SD-score.

Results: Of 144 children (75 males, mean age $6.5 \pm 4.5$ years), 52 (36\%) had an underlying chronic disease. According to STRONGkids, 46 (32\%) children were at low risk, 76 (53\%) at moderate risk and 22 (15\%) at high risk for malnutrition. The latter had significantly lower Height for Age values (mean SD value $-1.07 \pm 2.08 ; p=0.008$ ) and BMI values (mean SD-values $-0.79 \pm 2.09 ; p=0.0021$ ) in comparison to other groups. However, only 29 children were actually malnourished.

Conclusions: The STRONGkids is easy to administer. It is highly sensitive but not specific. It may be used as a very preliminary screening tool to be integrated with other clinical data in order to reliably predict the risk of malnutrition.
\end{abstract}

Keywords: Pediatrics, Children, Hospital malnutrition, Screening tool, Chronic disease

\section{Italian abstract}

Please see Additional file 1 for translation of the abstract into Italian language.

\section{Background}

Malnutrition is associated with negative outcomes for inpatients, including increased risks of infections [1,2], increased muscle loss [3], impaired wound healing, longer hospital stay and increased morbidity and mortality [4-6]. Malnutrition may be responsible for delayed recovery and need for intensive nursing care, thus increasing the cost of hospitalization [7].

Data on acute and chronic malnutrition of children admitted to hospital are strictly dependent on the criteria used for its definition [8]. Malnutrition rates from

\footnotetext{
* Correspondence: alfguari@unina.it

Department of Translational Medical Science - Section of Pediatrics, University of Naples "Federico II", Naples, Italy
}

6 to $19 \%$ have been reported in European countries such as UK, France, Germany and the Netherlands, reaching $40 \%$ in Turkey [9-13]. A recent Italian study evaluated the incidence of hospital-acquired malnutrition in 496 children admitted for diagnostic procedures, minor infections, or other episodic illness, and reported that children with a BMI Z-score $<-2$ SD on admission showed a mean BMI decrease at the end of their hospital stay that was significantly higher than those with a better nutritional condition at admission [14].

Assessment of nutritional status is not easy in pediatric practice and there is no single parameter to define malnutrition. Assessment of patients' actual nutritional status only identifies those who are already malnourished [15], while early identification of children at risk for malnutrition could promote timely nutritional interventions, preventing the short and long-term consequences of malnutrition. Routine screening for nutritional risk in children is
Ciomed Central

(c) 2013 Spagnuolo et al.; licensee BioMed Central Ltd. This is an open access article distributed under the terms of the Creative Commons Attribution License (http://creativecommons.org/licenses/by/2.0), which permits unrestricted use, distribution, and reproduction in any medium, provided the original work is properly cited. 
hampered by the lack of validated nutritional assessment protocols and evaluation of weight gain and growth velocity remains the standard method [16]. Several screening tools have been proposed to assess the risk of malnutrition but their application is hampered by the limited data and their acceptance for broad use [17]. A screening tool for nutritional risk in children, called Screening Tool for Risk Of impaired Nutritional status and Growth (STRONGkids), was successfully applied in the Netherlands [18]. Subsequently the STRONGkids was tested in patients with Intestinal Bowel Diseases (IBD) but its reliability was unclear $[19,20]$.

Aim of the present study was to investigate the efficacy of STRONGkids instrument in a population of children consecutively admitted to 12 Italian hospitals. Sensitivity, specificity and predictivity were assessed by comparing the scores of risk with the actual nutritional status of children. The scores of STRONGkids were also correlated with risk factors for malnutrition including age, the specific etiology for which the child was admitted to hospital and the association with underlying chronic diseases in order to examine their possible role.

\section{Materials and methods}

A prospective observational multi-centre study was performed in 12 hospitals in Campania region, Italy, (including one University hospital), covering virtually $70 \%$ of entire the pediatric population living in the Region, during the months of October-November 2012. Italian children from 1 to 18 years of age admitted to hospital for any disease, hence unselected were enrolled. Patients in intensive care were excluded. Reasons for admission were classified as infectious, gastrointestinal, respiratory, genetic/metabolic, neurological, oncological, trauma, surgical, cardiac, and others.

The STRONGkids consists of 4 items (1- high risk underlying disease, 2- clinical assessment, 3- nutritional intake and presence of vomit or diarrhea, 4- recent weight loss) and children are classified in one out of three classes for malnutrition (low, moderate, high risk) according to a specific 5-points scale (low $=0$, moderate $=$ $1-3$, high $=4-5)$. Each item is allocated a score of 1-2 points as follows:

- High risk disease (2 points): underlying illness with a risk of malnutrition or major surgery planned. The conditions that can lead to nutritional risk listed in the STRONGkids are: anorexia nervosa, congenital heart diseases, celiac disease, expected major surgery, dysmaturity/prematurity, bronchopulmonary dysplasia (maximum age 2 years), cystic fibrosis, digestive fistula, inflammatory bowel disease, infectious disease, metabolic disease, cancer, pancreatitis, chronic liver disease, muscle disease, chronic kidney disease, mental handicap/retardation, sepsis, short bowel syndrome, trauma, burns, other (specified by physician) [18].

- Subjective clinical assessment (1 point): poor nutritional status as judged by subjective clinical assessment (decreased subcutaneous fat and/or muscle mass and/or hollow face).

- Nutritional intake and losses (1 point): a) presence of diarrhoea with $\geq 5$ stools/day and/or vomiting with $>3$ times/day in the last few days or b) reduced food intake in the last few days before admission or c) advised nutritional intervention or d) inability to receive adequate colonic intake because of pain.

- Weight loss or poor weight gain (1 point): weight loss or no weight gain (infants $<1$ year) in the last few weeks/months.

The questionnaires were administered by a nurse on one predetermined day, thus including all patients admitted that day, and collected by the study coordinating nurse (DM).

Anthropometric measurements were taken at admission and compared with published standard values obtained in an Italian reference pediatric population [21]. The STRONGkids score of each child was compared with his/her actual nutritional status expressed as BMI SD-score and Height-for-Age (HFA) SD-score. SDscores $<-2$ for BMI and HFA were considered hallmarks of acute [22] and chronic malnutrition [23] respectively. Malnutrition rate was defined as the presence of acute and/or chronic malnutrition.

Informed consent was obtained from parents of enrolled children and the Ethics Committee of University of Naples 'Federico II' approved the protocol.

\section{Statistical analysis}

Data were expressed as number/percent or as mean \pm SD. Descriptive analyses were used to describe the study population and BMI was expressed as SD-score. Comparison of continuous data between groups was carried out using the $t$-test. The $\chi^{2}$ method, or the exact Fisher's test when appropriate, was applied to compare the presence of chronic conditions and the reasons for admission. Sensitivity, specificity, Positive Predictive Value (PPV) and Negative Predictive Value (NPV) of STRONGkids were calculated based on BMI and HFA SD-scores, using their cut-off values. Univariate regression analysis was applied to identify the main factors associated with the following outcomes of interest: acute, chronic and overall malnutrition and high risk class of malnutrition according to STRONGkids. P (two-sided) $<0.05$ was considered as significant. Data were analyzed with the SPSS package version 17.0. 
Table 1 General features of children

\begin{tabular}{lc}
\hline Patient characteristics & \\
\hline Sex, M:F (\%) & $52: 48$ \\
Mean age (years) \pm SD $(95 \% \mathrm{Cl})$ & $6,5 \pm 4,5(5.7-7.2)$ \\
Hospital, University: General (\%) & $42: 58$ \\
Mean SD-scores BMI \pm SD $(95 \% \mathrm{Cl})$ & $0.05 \pm 1.85(-0.2-0.3)$ \\
Mean SD-scores HFA $\pm S D(95 \% \mathrm{Cl})$ & $-0.37 \pm 1.84(-0.68-0.06)$ \\
Underlying diseases n/N (\%) & $52 / 144(36)$ \\
\hline
\end{tabular}

\section{Results}

\section{General features}

A total of 144 children (75 males mean age $6.5 \pm$ 4.5 years) were enrolled (Table 1 ). Sixty patients were admitted in a University Children's hospital and their data were recorded in 4 different days, whereas the other 84 were enrolled in 11 general hospitals in a single day of observation. Overall 52/144 (36\%) of the hospitalized children suffered from an underlying chronic disease (Table 1). One third of children were admitted for an infectious disease (43/144). Non-infectious gastrointestinal (such as IBD) or respiratory (such as asthma) conditions were other common etiologies of admission (Figure 1).

\section{STRONGkids scores and anthropometrics}

According to STRONGkids score, 46 (32\%) children were at low risk, 76 (53\%) at moderate risk and 22 (15\%) at high risk to develop malnutrition. Twenty-nine (20\%) children were malnourished according to BMI (16/144; $11 \%)$ and HFA SD-scores (15/144; 10\%), including 2 patients who met the criteria for both acute and chronic malnutrition (Table 2). However, only 5 of these 29 malnourished children (17\%) were classified at high risk by
STRONGkids. No difference was found in the incidence of acute and chronic malnutrition between risk classes. Mean SD-scores for HFA and BMI of the 144 children were $-0.37 \pm 1.85$ and $0.05 \pm 1.86$ respectively (Table 3 ). Children with high risk STRONGkids score had significantly lower SD-scores for BMI $(-0.79 \pm 2.09$; p $=0.002)$ and for HFA $(-1.07 \pm 2.08 ; \mathrm{p}=0.008)$ in comparison with other groups (Table 3).

A weak albeit significant linear correlation was found between the STRONGkids scores and anthropometric measurements, considering both BMI SD-scores $(r=-0.238$; $\mathrm{p}=0.0065)$ and HFA SD-scores $(\mathrm{r}=-0.311 ; \mathrm{p}=0.0002)$ (Figure 2a-b).

\section{Sensitivity, specificity and predictive values}

Scores in the range of medium plus high risk (1-5) identified children at risk for malnutrition with a $71 \%$ sensitivity (95\% CI: 48-89) and 53\% specificity (95\% CI: 4363) (LR 1.5; p = 0.032) based on anthropometrics. The positive predictive value of the same scores was $21 \%$ (95\% CI: 17-25) and their negative predictive value was 85\% (95\% CI: 85-90). Sensitivity, specificity and predictive values of the STRONGkids classes are provided in Table 3.

\section{Risk factors for malnutrition according to anthropometrics}

The role of putative risk factors for malnutrition was also evaluated by analyzing the distribution of selected variables (Table 4). Children $\leq 5$ years of age had a significantly higher risk to develop malnutrition (OR = 2.708; $\mathrm{p}=0.024)$, particularly acute malnutrition $(\mathrm{OR}=$ 4.602; $\mathrm{p}=0.006)$. The presence of underlying diseases

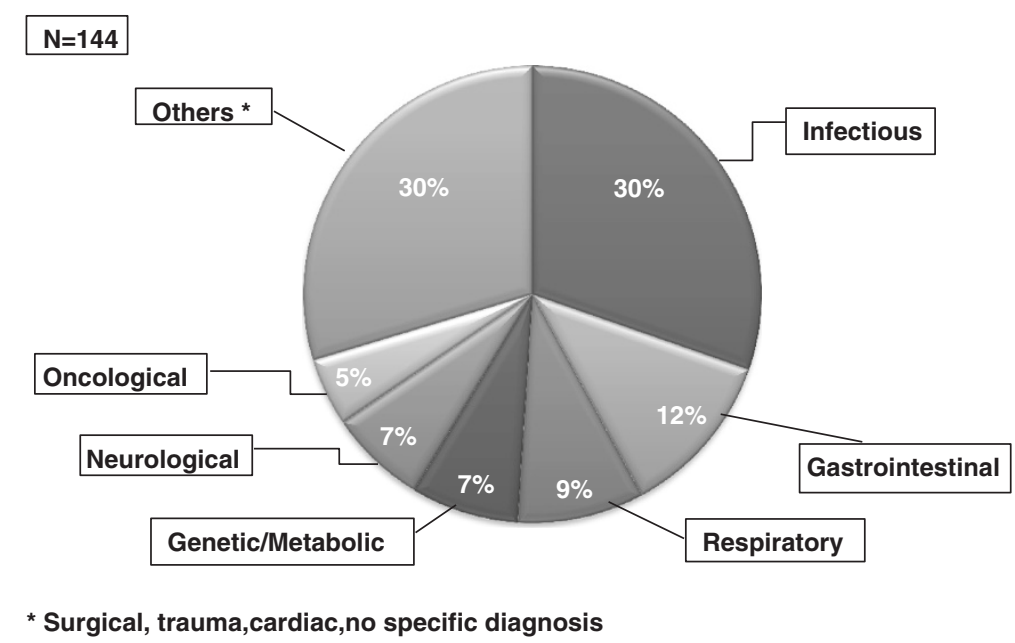

Figure 1 Etiology distribution of children according to hospital setting. The most common cause of admissions was an acute infectious disease. However, chronic gastrointestinal conditions, including Inflammatory Bowel Disease, and respiratory conditions were as common. Most heterogeneous conditions labelled as 'Others' were acute conditions. Therefore, overall chronic and acute conditions were equally represented in the observed population. 
Table 2 Distribution of children with acute and chronic malnutrition in the STRONGkids classes of risk for malnutrition

\begin{tabular}{|c|c|c|c|c|}
\hline & $\begin{array}{c}\text { Low } \\
(\mathrm{N}=46)\end{array}$ & $\begin{array}{l}\text { Moderate } \\
(\mathrm{N}=76)\end{array}$ & $\begin{array}{l}\text { High } \\
(\mathrm{N}=22)\end{array}$ & $\begin{array}{c}\text { Tot } \\
(\mathrm{N}=144)\end{array}$ \\
\hline Acute malnutrition (BMI SD-scores $<-2$ ) & $5(11 \%)$ & $9(12 \%)$ & $2(9 \%)$ & $16(11 \%)$ \\
\hline Chronic malnutrition (HFA SD-scores $<-2$ ) & $2(4 \%)$ & $9(12 \%)$ & $4(18 \%)$ & $15(10 \%)$ \\
\hline Overall malnutrition (Acute+Chronic) & $7(15 \%)$ & $17^{\mathrm{a}}(22 \%)$ & $5^{\mathrm{a}}(22 \%)$ & $29^{\mathrm{a}}(20 \%)$ \\
\hline
\end{tabular}

atwo patients were classified with both acute and chronic malnutrition.

also contributed to the development of malnutrition $(\mathrm{OR}=10.234 ; \mathrm{p}=0.036)$, and overall the etiology played a role: children with a diagnosis of genetic disease had a very significantly higher risk to develop chronic malnutrition $(\mathrm{OR}=10.167 ; \mathrm{p}=0.002)$ than other diagnostic groups. Furthermore, children with a diagnosis of gastrointestinal diseases (such as IBD) were more frequently classified at high nutritional risk by STRONGkids $(\mathrm{OR}=3.75$; $\mathrm{p}=0.026)$ (Table 4).

\section{Discussion}

A number of screening tools have been proposed and their features were recently reviewed [17]. Reliability (e.g. predictivity) and acceptance by both health care workers and patients are major factors for success of a specific tool. However, in our population of approximately 150 children in 12 different hospitals, a total of $70 \%$ were at moderate or high nutritional risk according to STRONGkids, but only approximately $20 \%$ were actually malnourished according to anthropometric measurements. Even if the STRONGkids has been designed to assess the risk and not the actual presence of malnutrition, the gap between the actual incidence and the estimated risk was substantial. It should be considered that the STRONGkids score assigns 2 points (out of the maximum total of 5) to a patient with a reported underlying illness, which is by itself sufficient to include him/her in the moderate class for risk. Although the presence of chronic disease is associated with high risk for complications during hospitalization [24,25], including malnutrition [26], when we looked at their distribution in the STRONGkids, several conditions with a putative intrinsic nutritional risk were not associated with malnutrition. For example, celiac disease (when adequately controlled) [27] or mental retardation, do not necessarily imply a nutritional risk, while congenital heart diseases is usually resolved within the first months of life, but its presence in the patient clinical history is sufficient to put the child in the high risk class for malnutrition.

We found a significant but weak correlation between the STRONGkids score and the parameters of acute and chronic malnutrition. According to our results, the correlation between the STRONGkids and HFA-SD scores (hallmark of chronic malnutrition) was slightly stronger than that between the STRONGkids and BMI-SD scores (index of acute malnutrition). This appears to be in contrast with another study [28] in which the STRONGkids was significantly related with both BMI and HFA. In the latter study the STRONGkids provided more reliable information compared to the STAMP (Screening Tool for the Assessment of Malnutrition in Pediatrics) [29]. A possible explanation is the different weight of the item "underlying disease" in the two scoring systems. The STRONGkids includes a large list of chronic conditions, while in the STAMP there is the generic question "Does the child have a diagnosis that has any nutritional implications?"

Significant correlations between anthropometric measurements and the STRONGkids' scores were found only for the high risk group, thus confirming that only children at high nutritional risk were actually already malnourished. Although less reliable compared to what reported in the Dutch study, the STRONGkids showed a high negative predictive value. In contrast the positive predictive value was low.

Table 3 STRONGkids classes of risk for malnutrition and mean anthropometric measurements

\begin{tabular}{lcccc}
\hline & $\begin{array}{c}\text { Low } \\
(\mathbf{N}=\mathbf{4 6})\end{array}$ & $\begin{array}{c}\text { Moderate } \\
\mathbf{( N = 7 6 )}\end{array}$ & $\begin{array}{c}\text { High } \\
\mathbf{( N = 2 2 )}\end{array}$ & $\begin{array}{c}\text { Tot } \\
\mathbf{( N = 1 4 4 )}\end{array}$ \\
\hline Mean SD-scores BMI \pm SD & $0.50 \pm 1.90$ & $0.02 \pm 1.66$ & $-0.79 \pm 2.09^{\mathrm{a}}$ & $0.05 \pm 1.86$ \\
Mean SD-scores HFA \pm SD & $0.26 \pm 1.19$ & $-0.58 \pm 2.01$ & $-1.07 \pm 2.08^{\mathrm{b}}$ & $-0.37 \pm 1.85$ \\
Sensitivity (95\% Cl) & $34 \%(25-43)$ & $71 \%(48-89)$ & $86 \%(78-92)$ & $71 \%(48-89)$ \\
Specificity (95\% Cl) & $75 \%(55-89)$ & $53 \%(43-63)$ & $21 \%(8-41)$ & $21 \%(17-25)$ \\
Positive predictive value (95\% Cl) & $9 \%(5-13)$ & $21 \%(17-25)$ & $28 \%(19-37)$ & $85 \%(85-90)$ \\
Negative predictive value $(95 \% \mathrm{Cl})$ & $73 \%(69-77)$ & $85 \%(85-90)$ & $82 \%(79-85)$ & \\
\hline
\end{tabular}

${ }^{a}$ Significantly lower SD-BMI compared to low and moderate categories $(p=.0021)$.

${ }^{\mathrm{b}}$ Significantly lower SD-HFA compared to low and moderate categories $(p=.008)$. 


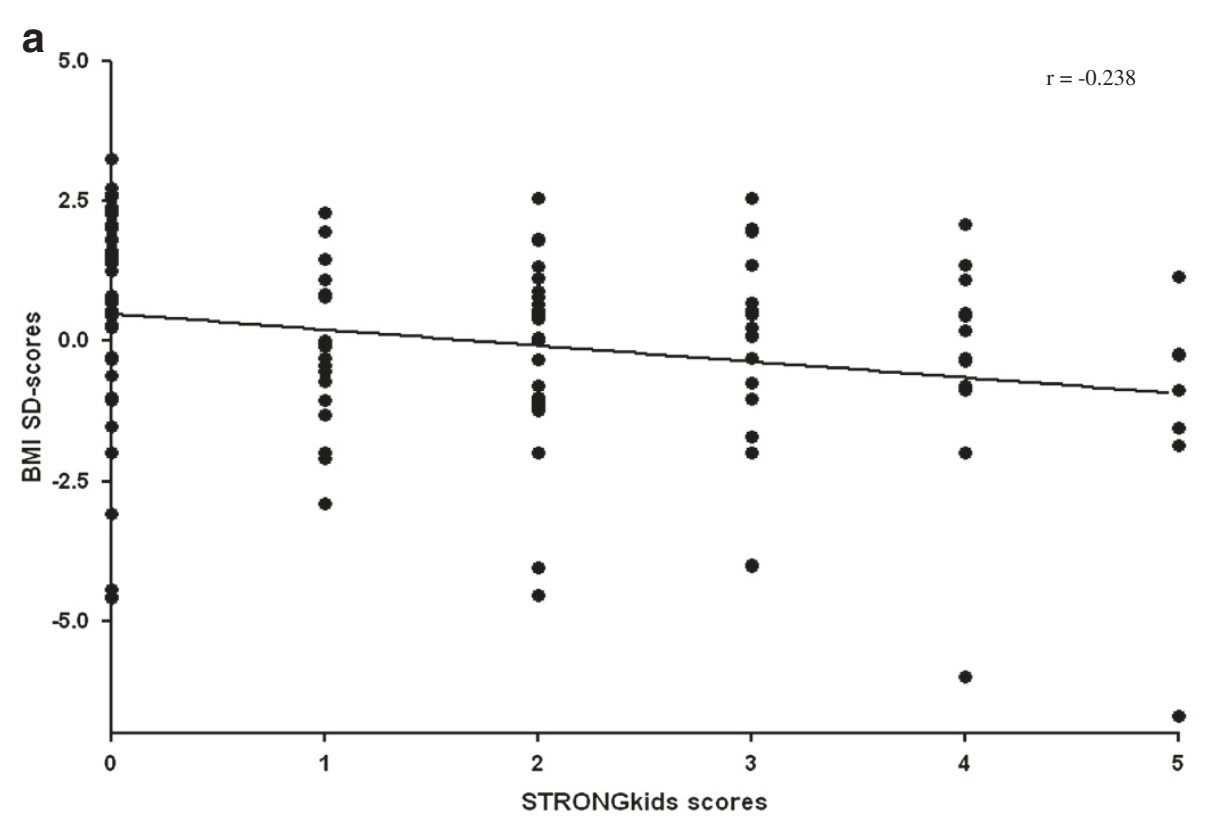

b

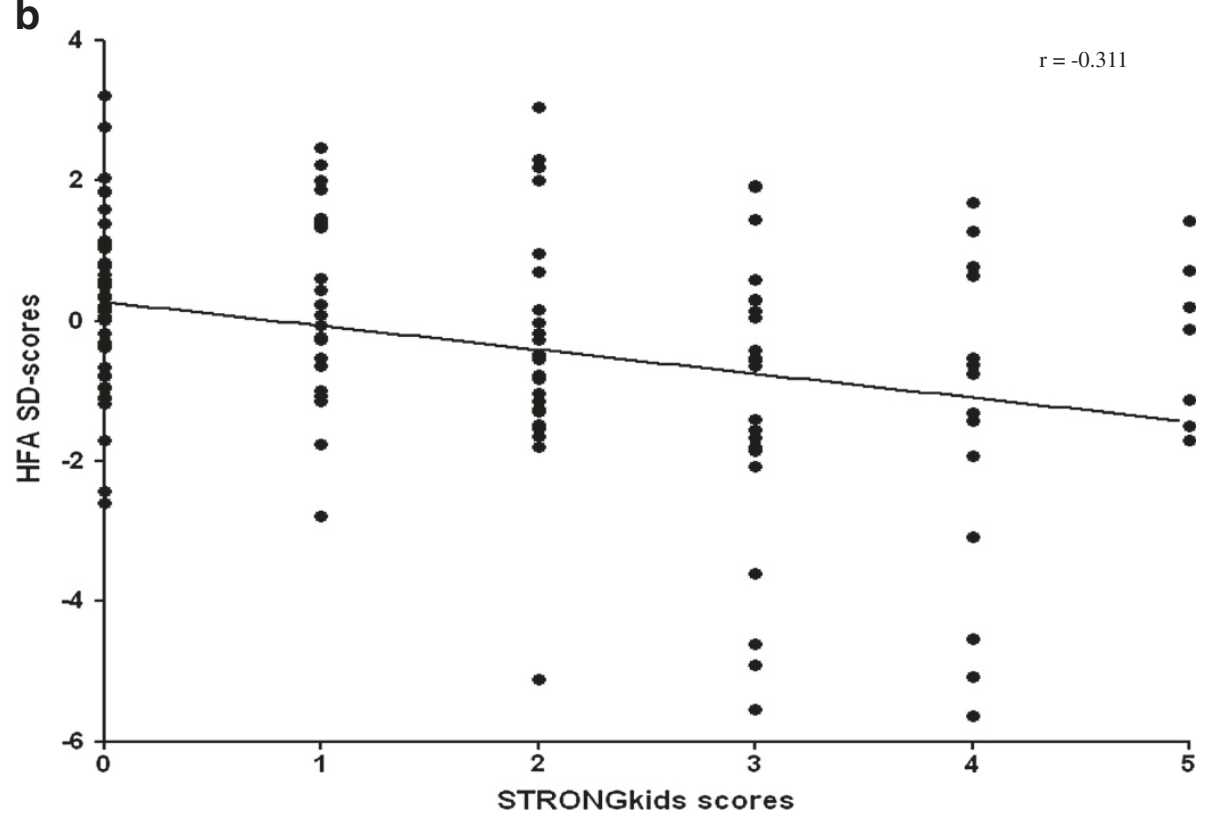

Figure 2 Linear correlation between anthropometric measurements and STRONGkids scores. (a) Correlation with BMI SD-SCores. (b) Correlation with Height-for-Age SD-scores.

Table 4 Risk factors for malnutrition

\begin{tabular}{|c|c|c|c|c|c|c|c|c|}
\hline \multirow[t]{2}{*}{ Determinant } & \multicolumn{2}{|c|}{$\begin{array}{l}\text { Acute malnutrition } \\
\text { (BMI SD-score <-2) }\end{array}$} & \multicolumn{2}{|c|}{$\begin{array}{l}\text { Chronic malnutrition } \\
\text { HFA SD-score }<-2\end{array}$} & \multicolumn{2}{|c|}{ Overall malnutrition } & \multicolumn{2}{|c|}{ High risk class } \\
\hline & $\begin{array}{c}\text { OR } \\
(95 \% \mathrm{Cl})\end{array}$ & $p$ & $\begin{array}{c}\text { OR } \\
(95 \% \mathrm{Cl})\end{array}$ & $p$ & $\begin{array}{c}\text { OR } \\
(95 \% \mathrm{Cl})\end{array}$ & $p$ & $\begin{array}{c}\text { OR } \\
(95 \% \mathrm{Cl})\end{array}$ & $p$ \\
\hline Age $\leq 5$ years & $4.602(1.43-14.77)$ & $.006^{\mathrm{a}}$ & $1.295(0.44-3.78)$ & .42 & $2.708(1.16-6.31)$ & $.024^{b}$ & $1.393(0.56-3.47)$ & .496 \\
\hline Any underlying disease & $1.841(0.68-4.97)$ & .297 & $2.18(0.72-6.19)$ & .257 & $1.04(1.01-2)$ & $.036^{c}$ & $10.234(3.76-28.91)$ & .001 \\
\hline Genetic disease & $2.925(0.7-12.24)$ & .145 & $10.167(2.63-39.24)$ & $.002^{d}$ & $8.293(2.24-30.68)$ & $.002^{\mathrm{e}}$ & $0.529(0.06-4.35)$ & .547 \\
\hline Gastrointestinal disease & $1.01(0.3-1.59)$ & .129 & $1.89(0.7-5.23)$ & .216 & $1.55(1.22-3.15)$ & $.023^{f}$ & $3.75(1.22-11.5)$ & $.026^{9}$ \\
\hline
\end{tabular}


When we examined the risk factors associated with the risk of malnutrition, an age $\leq 5$ years was associated with a higher risk of acute malnutrition, probably because younger children have a higher incidence of acute hospitalization-related malnutrition [30,31].

Specific etiologies were associated with a risk of malnutrition, including genetic/metabolic diseases. Also children with gastrointestinal diseases (especially those with IBD) were more likely to have scores in high risk class. A recent study in 46 patients with IBD showed the limits of several nutritional screening tools and pointed out that children with IBD are at high nutritional risk: many are underweight, even though the majority is of normal weight and some are overweight for their height [20]. We found similar results in our study.

We believe that the STRONGkids scores should be considered together with clinical and anthropometric data. In fact, many pediatricians who were involved in this study reported that the major limit of the instrument was the mismatch between their clinical judgement of patient's actual nutritional risk and the categorization (high, moderate or low) deriving from the STRONGkids assessment (data not shown).

Tools, such as the Subjective Global nutritional Assessment (SGA) proposed by Secker and Jeejeebhoy [32,33], include a specific evaluation of physical parameters, objectively looking at signs of fat and muscle wasting (i.e. edema). In contrast, the STRONGkids does not include an objective assessment and it should be regarded as a very preliminary screening to be collected in the history and integrated with other clinical data in order to reliably predict the risk of malnutrition.

Routine screening for nutritional risk in children is currently hampered by a lack of validated and easy methods for nutritional assessment. In addition to the STRONGkids, other tools were designed in order to evaluate nutritional risk, but each of them showed some limits. The "Simple Pediatric Nutritional Risk Score" [34] (SPNRS) and the SGA tools [32,33] are considered too complicated and time-consuming and consequently their uptake has been limited [17]. Gerasimidis et al. [35] developed the Paediatric Yorkhill Malnutrition Score (PYMS), which is a four-stage evaluation, considering the BMI value, recent weight loss, decreased intake in the previous week, and expected affected nutrition by the admission/condition for the next week. Nutrition screening by nurses using the new PYMS score is feasible for pediatric inpatients, identifies children at risk of malnutrition and efficiently uses available resources.

However, in a recent study the STRONGkids was compared to PYMS and STAMP and it was the only tool that recognized all undernourished children in its medium or high risk groups [36].

In conclusion, the main positive feature of STRONGkids consists in its simple structure which makes it easy to use in any hospital setting. However its reliability and efficacy are limited. STRONGkids effectively drives attention towards important issues related to nutritional risk. Probably the major limit is that the score appears affected by the high scores given to underlying diseases, that however are associated with a true nutritional risk only when they are "active". These limits might be improved with some modifications in the classification of patients, in order to identify children at actual nutritional risk. The main modification should be re-evaluation of the score given to chronic condition.

\section{Additional file}

Additional file 1: Italian abstract

\section{Abbreviations}

ART: Antiretroviral therapy; Cl: Confidence interval for data estimates; Crl: Credibility interval for model projections - defined as the 2.5 to $97.5 \%$ percentile range; DALY: Disability adjusted life year; DfID: Department for International Development; FSW: Female sex workers; HIV: Human immunodeficiency virus; HSV-2: Herpes simplex type 2; ICER: Incremental cost-effectiveness ratio; IPM: International Partnership for Microbicides; MDP: Microbicide development programme.

\section{Competing interests}

The authors declare that they have no competing interests.

\section{Authors' contribution}

MIS and AG conceived of and designed the study. DM collected all the data as coordinating nurse. MIS, IL and FC analyzed the data. MIS wrote the first draft of the manuscript. AG, IL and FC contributed to the writing of the manuscript. MIS, IL, FC, DM and AG agreed with the manuscript results. All authors read and approved the final manuscript.

\section{Acknowledgments}

We would like to thank all of our colleagues who participated in the enrolment of patients.

\section{Sources of funding}

The study was supported by Nutricia-Advanced Medical Nutrition.

Received: 29 August 2013 Accepted: 17 December 2013

Published: 27 December 2013

\section{References}

1. Kruizenga HM, Tulder MWV, Seidell JC: Effectiveness and cost-effectiveness of early screening and treatment of malnourished patients. Am J Clin Nutr 2005, 82:1082-1089

2. Baldwin C, Parson TJ: Dietary advice and nutrition supplements in the management of illness-related malnutrition: a systematic review. Clin Nutr 2004, 23:1267-1279.

3. Hoffer $\sqcup$ : Clinical Nutrition: 1. Protein-energy malnutrition in the inpatient. Can Med Assoc J 2001, 165:1345-1349.

4. Braunschweig C, Gomez S, Sheean PM: Impact of declines in nutritional status on outcomes in adult patients hospitalized for more than 7 days. J Am Diet Assoc 2000, 100:1316-1322.

5. Holmes S: The effects of undernutrition in hospitalised patients. Nurs Stand 2007, 22:35-38.

6. Kubrack $C$, Jensen L: Malnutrition in acute care patients. Int I Nurs Stud 2007, 44:1036-1054

7. Correia MI, Waitzberg DL: The impact of malnutrition on morbidity, mortality, length of hospital stay and costs evaluated through a multivariate model analysis. Clin Nutr 2003, 22:235-239.

8. Joosten KF, Hulst JM: Prevalence of malnutrition in pediatric hospital patients. Curr Opin Pediatr 2008, 20:590-596. 
9. Dogan $Y$, Erkan T, Yalvac $S$, et al: Nutritional status of patients hospitalized in pediatric clinic. Turk J Gastroenterol 2005, 16:212-216.

10. Marteletti $O$, Caldari D, Guimber D, et al: Malnutrition screening in hospitalized children: influence of the hospital unit on its management. Arch Pediatr 2005, 12:1226-1231.

11. Moy R, Smallman S, Booth I: Malnutrition in a UK children's hospital. J Hum Nutr Diet 1990, 3:93-100.

12. Pawellek I, Dokoupil K, Koletzko B: Prevalence of malnutrition in paediatric hospital patients. Clin Nutr 2008, 27:72-76.

13. Joosten KF, Zwart H, Hop WC, et al: National malnutrition screening days in hospitalized children in the Netherlands. Arch Dis Child 2010, 95:141-145.

14. Campanozzi A, Russo M, Catucci A, Rutigliano I, Canestrino G, Giardino I, Romondia A, Pettoello-Mantovani M: Hospital-acquired malnutrition in children with mild clinical conditions. Nutrition 2009, 25:540-547.

15. Aurangzeb $B$, Whitten KE, Harrison B, et al: Prevalence of malnutrition and risk of under-nutrition in hospitalized children. Clin Nutr 2012, 31:35-40.

16. Hartman C, Shamir R: Basic clinical assessment of pediatric malnutrition. Ann Nestle Eng 2009, 67:55-63.

17. Hartman C, Shamir R, Hecht C, Koletzko B: Malnutrition screening tools for hospitalized children. Curr Opin Clin Nutr Metab Care 2012, 15:303-309.

18. Hulst JM, Zwart H, Hop WC, et al: Dutch national survey to test the STRONGkids nutritional risk screening tool in hospitalized children. Clin Nutr 2010, 29:106-111.

19. Sandhu BK, Fell JME, Beattie RM: Guidelines for the management of inflammatory bowel disease in children in the United Kingdom. J Pediatr Gastroenterol Nutr 2010, 50(suppl1):S1-S13.

20. Wiskin AE, Owens DR, Cornelius VR, et al: Paediatric nutrition risk scores in clinical practice: children with inflammatory bowel disease. J Hum Nutr Diet 2012, 25:319-322.

21. Cacciari E, Milani S, Balsamo A, et al: Italian cross-sectional growth charts for height, weight and BMI (2 to $20 \mathrm{yr}$ ). J Endocrinol Invest 2006, 29:581-593.

22. Cole TJ, Flegal KM, Nicholls D, et al: Body mass index cut offs to define thinness in children and adolescents: international survey. BMJ 2007, 335:194.

23. WHO: Management of severe malnutrition: a manual for physicians and other senior health workers. Geneva: World Health Organization; 1999.

24. Skillman HE, Wischmeyer PE: Nutrition therapy in critically ill infants and children. J Parenteral Enteral Nutr 2008, 32:520-534

25. López-Herce Cid J, Sánchez Sánchez C, Mencía Bartolomé S, et al: Energy expenditure in critically ill children: correlation with clinical characteristics, caloric intake, and predictive equations. An Pediatr (Barc) 2007, 66:229-239.

26. Mosby $\Pi$ : Almost a fifth of children admitted to Dutch hospitals have acute or chronic malnutrition; risk factors include underlying disease and non-white ethnicity. Evid Based Nurs 2010, 13:81-82.

27. Barton SH, Kelly DG, Murray JA: Nutritional deficiencies in celiac disease. Gastroenterol Clin North Am 2007, 36:93-108.

28. Ling RE, Hedges $V$, Sullivan PB: Nutritional risk in hospitalized children: an assessment of two instruments. E-SPEN 2011, 6:e153-e157.

29. McCarthy $H$, Dixon M, Crabtree I, et al: The development and evaluation of the Screening Tool for the Assessment of Malnutrition in Paediatrics (STAMP@) for use by healthcare staff. J Hum Nutr Diet 2012, 25:311-318.

30. Hendricks KM, Duggar C, Gallagher L, et al: Malnutrition in hospitalized pediatric patients. Current prevalence. Arch Pediatr Adolesc Med 1995, 149:1118-1122.

31. Joosten KF, Hulst JM: Malnutrition in pediatric hospital patients: current issues. Nutrition 2011, 27:133-137.

32. Secker DJ, Jeejeebhoy KN: Subjective global nutritional assessment for children. Am J Clin Nutr 2007, 85:1083-1089.

33. Secker DJ, Jeejeebhoy KN: How to perform Subjective Global Nutritional assessment in children. J Acad Nutr Diet 2012, 112:424-431.

34. Sermet-Gaudelus I, Poisson-Salomon AS, Colomb V: Simple pediatric nutritional risk score to identify children at risk of malnutrition. Am J Clin Nutr 2000, 72:64-70.
35. Gerasimidis K, Keane O, Macleod I, et al: A four-stage evaluation of the Paediatric Yorkhill Malnutrition Score in a tertiary paediatric hospital and a district general hospital. Br J Nutr 2010, 19:1-6.

36. Modena V, Walls T, Day AS: Nutritional status and nutrition risk screening in hospitalized children in New Zealand. Acta Paediatr 2013, 102:e419-e423.

doi:10.1186/1824-7288-39-81

Cite this article as: Spagnuolo et al:: Application of a score system to evaluate the risk of malnutrition in a multiple hospital setting. Italian Journal of Pediatrics 2013 39:81.

\section{Submit your next manuscript to BioMed Central and take full advantage of:}

- Convenient online submission

- Thorough peer review

- No space constraints or color figure charges

- Immediate publication on acceptance

- Inclusion in PubMed, CAS, Scopus and Google Scholar

- Research which is freely available for redistribution

Submit your manuscript at www.biomedcentral.com/submit
C Biomed Central 